ESAIM: PROCEEDINGS, October 2011, Vol. 32, p. 1-17

E. Cancès, N. Crouseilles, H. Guillard, B. Nkonga, and E. Sonnendrücker, Editors

\title{
NUMERICAL SIMULATIONS OF THE FOCAL SPOT GENERATED BY A SET OF LASER BEAMS : LMJ *,**
}

\author{
Antoine Bourgeade ${ }^{1}$ And Boniface NkOnga ${ }^{2}$
}

\begin{abstract}
In order to get the fusion of small capsules containing a deuterium-tritium nuclear fuel, the MegaJoule laser (LMJ) will focus a large number of laser beams inside a cylinder (Hohlraum) which contains the fusion capsule. In order to control this process we have to know as well as possible the electromagnetic field created by the laser beams on both Hohlraum's apertures. This article describes a numerical tool which computes this electromagnetic field from the definition of the laser beams in front of the focusing gratings. Despite its apparent simplicity, this computation necessitates a two step plane wave decomposition and the size of the data requires the use of a parallel strategy with a large number of processors.

Résumé. Afin d'obtenir la fusion de petites cibles contenant du deuterium-tritium, le laser MégaJoule doit focaliser un grand nombre de faisceaux laser à l'intérieur d'un cylindre (Hohlraum) contenant une de ces cibles. La maîtrise de ce processus nécessite une bonne connaissance du champ électromagnétique induit par les faisceaux laser sur chacune des ouvertures du Hohlraum. Cet article décrit l'outil numérique mis au point pour le calcul de ce champ à partir de la description des faisceaux laser avant la focalisation. La simplicité du calcul n'est qu'apparente, puisque celui-ci a besoin d'une décomposition en ondes planes en deux étapes et que la taille des données nécessite l'utilisation d'un grand nombre de processeurs.
\end{abstract}

\section{INTRODUCTION}

The laser MegaJoule (LMJ) will have a large number of chains. Each chain is joining two sets of four laser beams, sets designed below as quads. Each quad of laser beams will be focused on a fusion target. The beams will cross through one of the two openings of the Hohlraum chamber, before propagating in the plasma and interacting with the inner surface of the cylinder. It is therefore important to have an accurate description of the total electromagnetic field resulting from the superposition of the LMJ laser beams at the openings of the Hohlraum. This total field is necessary to compute the subsequent plasma evolution. On the other hand, the total field can help to quantify the influence of some laser characteristics, such as the timing of the beams, smoothing, or reducing the FM/AM effect. Indeed, it is not the total instantaneous local field that is directly affecting the interaction physics but its statistical properties as the size of the focal spot size or density of hot spots or their average length. The problem seems as beams are propagating in a vacuum and their interference is described by a linear equation. The difficulty stems mainly from two facts. First the input face of Hohlraum

\footnotetext{
* This work was supported in part by CEA/CESTA, France

** Jean-Paul Goossens is acknowledged for helpful discussions.

1 CEA/CESTA, B.P. 2, 33114 Le Barp, France, e-mail: Antoine.Bourgeade@cea.fr

2 Department of Mathematics, University of Nice, France, e-mail: Boniface.Nkonga@unice.fr
} 
is not perpendicular to the axis of the beams and therefore does not correspond to the focal plane. Thus, the paraxial approximation that is defining the beam structure in the focal plane by the Fourier transform of the field before passing through the focusing system can not be used a priori. Secondly, given the size of the beams, the phase introduced by the gratings varies increasingly regarded as the point moves away from the centroid of the beams. This requires a high resolution in the Fourier space or equivalently a very fine mesh.

The ultimate goal of this work is to obtain a spatial and temporal description of the total field. We developed an efficient numerical approach for the calculation of the laser field obtained after focusing and overlapping of all quads (Figure 1) contributions on one side of the target (Figure 2). The purpose is to define an efficient numerical approach to compute the total spot electric field created by a set of laser beams(quads). This will allow at the LMJ facility to control the target irradiation and to achieve a symmetric implosion.

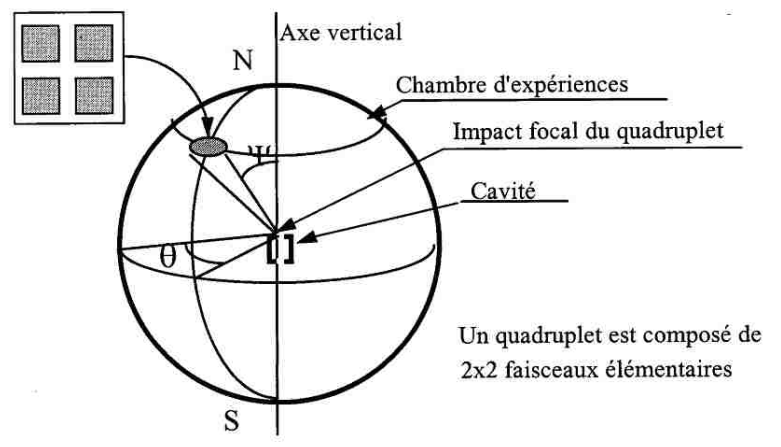

\begin{tabular}{|c|c|c|c|c|c|c|c|c|c|c|}
\hline Ycône & $\theta_{1}$ & $\theta 2$ & $\theta 3$ & $\theta 4$ & $\theta 5$ & $\theta 6$ & $\theta 7$ & $\theta 8$ & $\theta 9$ & $\theta 10$ \\
\hline $33^{\circ} 2$ & $0^{\circ}$ & $36^{\circ}$ & $72^{\circ}$ & $108^{\circ}$ & $144^{\circ}$ & $180^{\circ}$ & $216^{\circ}$ & $252^{\circ}$ & $288^{\circ}$ & $324^{\circ}$ \\
\hline $49^{\circ}$ & $18^{\circ}$ & $54^{\circ}$ & $90^{\circ}$ & $126^{\circ}$ & $162^{\circ}$ & $198^{\circ}$ & $234^{\circ}$ & $270^{\circ}$ & $306^{\circ}$ & $342^{\circ}$ \\
\hline $59^{\circ} 5$ & $0^{\circ}$ & $36^{\circ}$ & $72^{\circ}$ & $108^{\circ}$ & $144^{\circ}$ & $180^{\circ}$ & $216^{\circ}$ & $252^{\circ}$ & $288^{\circ}$ & $324^{\circ}$ \\
\hline $78^{\circ}$ & $18^{\circ}$ & $54^{\circ}$ & $90^{\circ}$ & $126^{\circ}$ & $162^{\circ}$ & $198^{\circ}$ & $234^{\circ}$ & $270^{\circ}$ & $306^{\circ}$ & $342^{\circ}$ \\
\hline $102^{\circ} 5$ & $0^{\circ}$ & $36^{\circ}$ & $72^{\circ}$ & $108^{\circ}$ & $144^{\circ}$ & $180^{\circ}$ & $216^{\circ}$ & $252^{\circ}$ & $288^{\circ}$ & $324^{\circ}$ \\
\hline $120^{\circ} 5$ & $18^{\circ}$ & $54^{\circ}$ & $90^{\circ}$ & $126^{\circ}$ & $162^{\circ}$ & $198^{\circ}$ & $234^{\circ}$ & $270^{\circ}$ & $306^{\circ}$ & $342^{\circ}$ \\
\hline $131^{\circ}$ & $0^{\circ}$ & $36^{\circ}$ & $72^{\circ}$ & $108^{\circ}$ & $144^{\circ}$ & $180^{\circ}$ & $216^{\circ}$ & $252^{\circ}$ & $288^{\circ}$ & $324^{\circ}$ \\
\hline $146^{\circ} 8$ & $18^{\circ}$ & $54^{\circ}$ & $90^{\circ}$ & $126^{\circ}$ & $162^{\circ}$ & $198^{\circ}$ & $234^{\circ}$ & $270^{\circ}$ & $306^{\circ}$ & $342^{\circ}$ \\
\hline
\end{tabular}

Figure 1. Description of quads. A quad is a set of four beams which are parallel and centered on the vertices of a square orthogonal to the direction of beam propagation. Specified angles define the propagation axis. In practice, only the first three angles of incidence are used, with the last three corresponding to the opposite side.

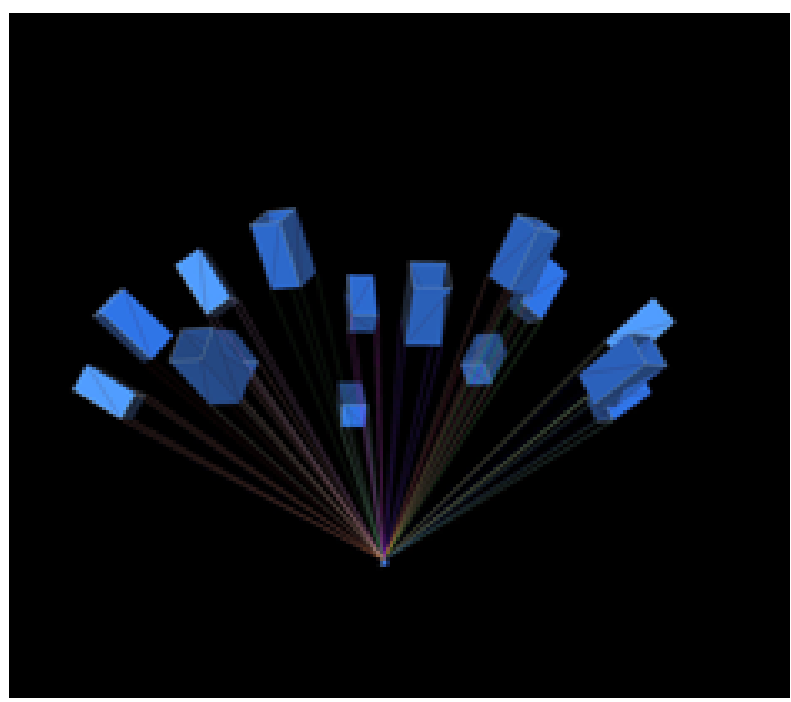

Figure 2. On side focusing configuration with 15 quads 


\section{MODEL}

A single laser beam, identified with the index $\ell$, is defined by a plane centered at the vertex $\boldsymbol{x}_{\ell}$ and orthogonal to the propagation direction $\boldsymbol{n}_{0, \ell}$ and the electric field $\mathcal{E}_{\ell}\left(\boldsymbol{\xi}_{\ell}, t\right)$ on this plane, where $\boldsymbol{\xi}_{\ell} \in \mathbb{R}^{2}$ is the coordinate associated to the plane with $\boldsymbol{\xi}_{\ell}\left(\boldsymbol{x}_{\ell}\right)=0$. For any point $\boldsymbol{x}$ in the space, we define by $\boldsymbol{\beta}_{\ell}(\boldsymbol{x})$ the coordinate related to the following beam as

$$
\boldsymbol{\beta}_{\ell}(\boldsymbol{x})=\boldsymbol{x}-\boldsymbol{x}_{\ell}=z_{\ell}(\boldsymbol{x}) \boldsymbol{n}_{0, \ell}+\xi_{1, \ell}(\boldsymbol{x}) \boldsymbol{n}_{1, \ell}+\xi_{2, \ell}(\boldsymbol{x}) \boldsymbol{n}_{2, \ell} \quad \text { for } \quad \boldsymbol{x} \in \Omega,
$$

where the set $\left(\boldsymbol{n}_{0, \ell}, \boldsymbol{n}_{1, \ell}, \boldsymbol{n}_{2, \ell}\right)$ is an orthogonal basis and $\Omega$ the focal spot. We also define spatial coordinates $\boldsymbol{\xi}_{\ell}$ and spectral coordinates $\boldsymbol{\theta}_{\ell}$ in the transverse direction of the laser propagation:

$$
\boldsymbol{\xi}_{\ell}=\left(\begin{array}{c}
\xi_{1, \ell} \\
\xi_{2, \ell}
\end{array}\right) \in \mathbb{R}^{2}, \quad \text { and } \quad \boldsymbol{\theta}_{\ell}=\left(\begin{array}{c}
\theta_{1, \ell} \\
\theta_{2, \ell}
\end{array}\right) \in \mathbb{R}^{2}
$$

In the present case, the beam propagates in vacuum so that the electric field $\boldsymbol{E}$ satisfies the Maxwell's equation:

$$
\frac{\partial^{2} \boldsymbol{E}}{\partial t^{2}}-c^{2}\left(\frac{\partial^{2} \boldsymbol{E}}{\partial x^{2}}+\frac{\partial^{2} \boldsymbol{E}}{\partial y^{2}}+\frac{\partial^{2} \boldsymbol{E}}{\partial z^{2}}\right)=0
$$

Therefore, the dispersion of any beam is obtained by the Fourier transforms in space and time:

$$
-\left(\omega^{2}-c^{2} \boldsymbol{k}_{\ell} \cdot \boldsymbol{k}_{\ell}\right) \widehat{\boldsymbol{E}}_{\ell}=0 \Longrightarrow \omega^{2}-c^{2} \boldsymbol{k}_{\ell} \cdot \boldsymbol{k}_{\ell}=0
$$

where $\omega$ is the laser frequency and $\boldsymbol{k}_{\ell} \in \mathbb{R}^{3}$. It is convenient to define $\boldsymbol{k}_{\ell}$ as

$$
\boldsymbol{k}_{\ell}=k_{z, \ell} \boldsymbol{n}_{0, \ell}+\theta_{1, \ell} \boldsymbol{n}_{1, \ell}+\theta_{2, \ell} \boldsymbol{n}_{2, \ell}
$$

Therefore the dispersion relation gives

$$
k_{z, \ell}^{2}\left(\boldsymbol{\theta}_{\ell}, \omega\right)=\frac{\omega^{2}}{c^{2}}-\theta_{1, \ell}^{2}-\theta_{2, \ell}^{2} \Longrightarrow k_{z, \ell}\left(\boldsymbol{\theta}_{\ell}, \omega\right)= \pm \sqrt{\frac{\omega^{2}}{c^{2}}-\boldsymbol{\theta}_{\ell} \cdot \boldsymbol{\theta}_{\ell}} .
$$

Let us apply the Fourier transform in time and in the transverse direction (defined by vectors $\boldsymbol{n}_{1, \ell}$ and $\boldsymbol{n}_{2, \ell}$ ) to equation (1). Then the following equation describes the field evolution along the coordinate $z_{\ell}$ associated to the direction $\boldsymbol{n}_{0, \ell}$ :

$$
-\frac{d^{2} \widehat{\boldsymbol{E}}_{\ell}}{d z_{\ell}^{2}}-\left(\frac{\omega^{2}}{c^{2}}-\boldsymbol{\theta}_{\ell} \cdot \boldsymbol{\theta}_{\ell}\right) \widehat{\boldsymbol{E}}_{\ell}=0 \Longleftrightarrow \frac{d^{2} \widehat{\boldsymbol{E}}_{\ell}}{d z_{\ell}^{2}}+\left(k_{z, \ell}^{2}\left(\boldsymbol{\theta}_{\ell}, \omega\right)\right) \widehat{\boldsymbol{E}}_{\ell}=0
$$

Then, given $\widehat{\boldsymbol{E}}_{\ell}$ for $z_{\ell}=0$ and assuming forward wave in the direction $\boldsymbol{n}_{0, \ell}$, we can compute

$$
\widehat{\boldsymbol{E}}_{\ell} \equiv \widehat{\boldsymbol{E}}_{\ell}\left(\boldsymbol{\theta}_{\ell}, \omega, z_{\ell}\right)=\widehat{\mathcal{E}}_{\ell}\left(\boldsymbol{\theta}_{\ell}, \omega\right) \exp \left(\imath k_{z, \ell} z_{\ell}\right), \quad \forall z_{\ell} \geq 0,
$$

where $\widehat{\mathcal{E}}_{\ell}\left(\boldsymbol{\theta}_{\ell}, \omega\right) \equiv \widehat{\boldsymbol{E}}_{\ell}\left(\boldsymbol{\theta}_{\ell}, \omega, z_{\ell}=0\right)$ is the Fourier transform of the incoming wave $\mathcal{E}_{\ell}\left(\boldsymbol{\xi}_{\ell}, t\right)$.

Inverse Fourier transform is used to compute the laser beam field at this point :

$$
\begin{aligned}
\boldsymbol{E}_{\ell}(t, \boldsymbol{x}) & =\iint_{\widehat{\widehat{\boldsymbol{c}}}_{\ell}} \exp \left[\imath\left(\boldsymbol{\theta}_{\ell} \cdot \boldsymbol{\xi}_{\ell}(\boldsymbol{x})-\omega t\right)\right] \widehat{\boldsymbol{E}}_{\ell}\left(\boldsymbol{\theta}_{\ell}, \omega, z_{\ell}\right) d \boldsymbol{\theta}_{\ell} d \omega \\
& =\iint_{\widehat{\mathcal{c}}_{\ell}} \exp \left[\imath\left(\boldsymbol{\theta}_{\ell} \cdot \boldsymbol{\xi}_{\ell}(\boldsymbol{x})+z_{\ell} k_{z, \ell}\left(\boldsymbol{\theta}_{\ell}, \omega\right)-\omega t\right)\right] \widehat{\mathcal{E}}_{\ell}\left(\boldsymbol{\theta}_{\ell}, \omega\right) d \boldsymbol{\theta}_{\ell} d \omega \\
& =\iint_{\widehat{\boldsymbol{c}}_{\ell}} \exp \left[\imath\left(\boldsymbol{k}_{\ell}\left(\boldsymbol{\theta}_{\ell}, \omega\right) \cdot \boldsymbol{\beta}_{\ell}(\boldsymbol{x})-\omega t\right)\right] \widehat{\mathcal{E}}_{\ell}\left(\boldsymbol{\theta}_{\ell}, \omega\right) d \boldsymbol{\theta}_{\ell} d \omega
\end{aligned}
$$


where $\widehat{\boldsymbol{C}}_{\ell}$ is the domain of definition (in the phase space) of the initial beam. The last point of the modeling is to define the incoming electric field. In the present paper, this field is the one obtained after a focusing gratings. It is defined by the Fourier transform in time as

$$
\widetilde{\mathcal{E}}_{\ell}\left(\boldsymbol{\xi}_{\ell}, \omega\right)=\mathcal{A}_{\ell}\left(\boldsymbol{\xi}_{\ell}, \omega\right) \exp \left[-\imath \frac{\omega}{c} \varphi_{\ell}\left(\boldsymbol{\xi}_{\ell}, \omega\right)\right]
$$

where $\mathcal{A}_{\ell}\left(\boldsymbol{\xi}_{\ell}, \omega\right)$ described the laser beam before the gratings and $\varphi_{\ell}\left(\boldsymbol{\xi}_{\ell}, \omega\right)$ is the phase change associated to the focusing gratings. Then we can compute the Fourier transform $\widehat{\mathcal{E}}_{\ell}\left(\boldsymbol{\theta}_{\ell}, \omega\right)$ as

$$
\widehat{\mathcal{E}}_{\ell}\left(\boldsymbol{\theta}_{\ell}, \omega\right)=\int_{\mathcal{C}_{\ell}} \mathcal{A}_{\ell}\left(\boldsymbol{\xi}_{\ell}, \omega\right) \exp \left[-\imath \frac{\omega}{c} \varphi_{\ell}\left(\boldsymbol{\xi}_{\ell}, \omega\right)\right] \exp \left[-\imath\left(\boldsymbol{\theta}_{\ell} \cdot \boldsymbol{\xi}_{\ell}\right)\right] d \boldsymbol{\xi}_{\ell}
$$

where $\mathcal{C}_{\ell}$ is the domain of definition (in the physical space) of the initial beam. In order to estimate this integral we use the fact that, the Fourier transform of a product is the convolution of Fourier transforms :

$$
\mathcal{F}\left[\mathcal{A}_{\ell}\left(\boldsymbol{\xi}_{\ell}, \omega\right) \exp \left[-\imath \frac{\omega}{c} \varphi_{\ell}\left(\boldsymbol{\xi}_{\ell}, \omega\right)\right]\right]=\mathcal{F}\left[\mathcal{A}_{\ell}\left(\boldsymbol{\xi}_{\ell}, \omega\right)\right] * \mathcal{F}\left[\exp \left[-\imath \frac{\omega}{c} \varphi_{\ell}\left(\boldsymbol{\xi}_{\ell}, \omega\right)\right]\right]
$$

However the function $\exp \left[-\imath \frac{\omega}{c} \varphi_{\ell}\left(\xi_{\ell}, \omega\right)\right]$ is not integrable, its modulus is equal to one and it strongly oscillates. To overcome this difficulty, let us consider the following integrable function

$$
\Phi_{\varepsilon}\left(\boldsymbol{\xi}_{\ell}, \omega\right)=\exp \left[-\varepsilon \boldsymbol{\xi}_{\ell} \cdot \boldsymbol{\xi}_{\ell}-\imath \frac{\omega}{c} \varphi_{\ell}\left(\boldsymbol{\xi}_{\ell}, \omega\right)\right]
$$

where $\varepsilon>0$ is a small parameter that will be set to zero to recover the original estimation.

$$
\widehat{\Phi}_{\varepsilon}\left(\boldsymbol{\theta}_{\ell}, \omega\right)=\int_{\boldsymbol{C}_{\ell}} \exp \left[-\varepsilon \boldsymbol{\xi}_{\ell} \cdot \boldsymbol{\xi}_{\ell}-\imath \frac{\omega}{c} \varphi_{\ell}\left(\boldsymbol{\xi}_{\ell}, \omega\right)\right] \exp \left(-\imath\left(\boldsymbol{\theta}_{\ell} \cdot \boldsymbol{\xi}_{\ell}\right)\right) d \boldsymbol{\xi}_{\ell}
$$

On the other hand we assume the following phase profile

$$
\frac{\omega}{c} \varphi_{\ell}\left(\boldsymbol{\xi}_{\ell}, \omega\right) \simeq \mu \frac{\boldsymbol{\xi}_{\ell} \cdot \boldsymbol{\xi}_{\ell}}{2}, \quad \mu \in \mathbb{R}
$$

For the target applications, we can use such a profile with $\mu=\frac{\omega_{*}}{c f_{\ell}}$, where $f_{\ell}$ is the focal length, and $\omega_{*}$ the frequency for which the focusing gratings have been defined. Then

$$
\Phi_{\varepsilon}\left(\boldsymbol{\xi}_{\ell}, \omega\right)=\exp \left[-\left(\varepsilon+\imath \frac{\mu}{2}\right) \boldsymbol{\xi}_{\ell} \cdot \boldsymbol{\xi}_{\ell}\right]
$$

The scaling property of Fourier transform reads:

$$
\text { if } g(t)=\Phi\left(\frac{t}{\alpha}\right) \quad \text { then } \quad \widehat{g}(\omega)=|\alpha| \widehat{\Phi}(\alpha \omega) \quad \forall \alpha \in \mathbb{R}^{*}
$$

Moreover, $\forall \alpha \in \mathbb{C}$ with a strictly positive real part, one obtains:

$$
\text { if } g(t)=\exp \left(-\alpha t^{2}\right) \text { then } \widehat{g}(\omega)=\sqrt{\frac{\pi}{\alpha}} \exp \left(-\frac{\omega^{2}}{4 \alpha}\right)
$$


In the present case we use these formulas, in two spatial dimensions, $\boldsymbol{\xi}_{\ell} \in \mathbb{R}^{d}(d=2)$

$$
\text { if } g\left(\boldsymbol{\xi}_{\ell}\right)=\exp \left(-\alpha \boldsymbol{\xi}_{\ell} \cdot \boldsymbol{\xi}_{\ell}\right) \quad \text { then } \quad \widehat{g}\left(\boldsymbol{\theta}_{\ell}\right)=\left(\frac{\pi}{\alpha}\right)^{\frac{d}{2}} \exp \left(-\frac{\boldsymbol{\theta}_{\ell} \cdot \boldsymbol{\theta}_{\ell}}{4 \alpha}\right) .
$$

We apply this formula with $\alpha_{\varepsilon}=\varepsilon+\imath \frac{\mu}{2}$ to obtain

$$
\widehat{\Phi}_{\varepsilon}\left(\boldsymbol{\theta}_{\ell}, \omega\right)=\int_{\mathcal{C}_{\ell}} \exp \left[-\left(\varepsilon+\imath \frac{\mu}{2}\right) \boldsymbol{\xi}_{\ell} \cdot \boldsymbol{\xi}_{\ell}\right] \exp \left(-\imath\left(\boldsymbol{\theta}_{\ell} \cdot \boldsymbol{\xi}_{\ell}\right)\right) d \boldsymbol{\xi}_{\ell}=\frac{\pi}{\varepsilon+\imath \frac{\mu}{2}} \exp \left(-\frac{\boldsymbol{\theta}_{\ell} \cdot \boldsymbol{\theta}_{\ell}}{4\left(\varepsilon+\imath \frac{\mu}{2}\right)}\right)
$$

and, taking the limit of $\varepsilon$ going to zero, we get

$$
\widehat{\Phi}\left(\boldsymbol{\theta}_{\ell}, \omega\right)=\frac{\pi}{\imath \frac{\mu}{2}} \exp \left(-\frac{\boldsymbol{\theta}_{\ell} \cdot \boldsymbol{\theta}_{\ell}}{4\left(\imath \frac{\mu}{2}\right)}\right)=-\imath \frac{2 \pi}{\mu} \exp \left(\imath \frac{1}{\mu} \frac{\boldsymbol{\theta}_{\ell} \cdot \boldsymbol{\theta}_{\ell}}{2}\right)
$$

Then

$$
\begin{aligned}
\widehat{\mathcal{E}}_{\ell}\left(\boldsymbol{\theta}_{\ell}, \omega\right) & =\int_{\widehat{\boldsymbol{c}}_{\ell}} \widehat{\mathcal{A}}_{\ell}\left(\boldsymbol{\zeta}_{\ell}, \omega\right) \widehat{\Phi}\left(\boldsymbol{\theta}_{\ell}-\boldsymbol{\zeta}_{\ell}, \omega\right) d \boldsymbol{\zeta}_{\ell} \\
& =\exp \left(\imath \frac{1}{\mu} \frac{\boldsymbol{\theta}_{\ell} \cdot \boldsymbol{\theta}_{\ell}}{2}\right) \int_{\widehat{\boldsymbol{c}}_{\ell}} \widehat{\mathcal{A}}_{\ell}\left(\boldsymbol{\zeta}_{\ell}, \omega\right) \widehat{\Phi}\left(\boldsymbol{\zeta}_{\ell}, \omega\right) \exp \left(-\imath \frac{1}{\mu} \boldsymbol{\theta}_{\ell} \cdot \boldsymbol{\zeta}_{\ell}\right) d \boldsymbol{\zeta}_{\ell} \\
& =\exp \left(\imath \frac{1}{\mu} \frac{\boldsymbol{\theta}_{\ell} \cdot \boldsymbol{\theta}_{\ell}}{2}\right) \int_{\widehat{\boldsymbol{c}}_{\ell}} \widehat{\mathcal{H}}_{\ell}\left(\boldsymbol{\zeta}_{\ell}, \omega\right) \exp \left(-\imath \frac{1}{\mu} \boldsymbol{\theta}_{\ell} \cdot \boldsymbol{\zeta}_{\ell}\right) d \boldsymbol{\zeta}_{\ell}=\exp \left(\imath \frac{1}{\mu} \frac{\boldsymbol{\theta}_{\ell} \cdot \boldsymbol{\theta}_{\ell}}{2}\right) \mathcal{H}_{\ell}\left(\frac{\boldsymbol{\theta}_{\ell}}{\mu}, \omega\right)
\end{aligned}
$$

where $\widehat{\mathcal{H}}_{\ell}\left(\boldsymbol{\zeta}_{\ell}, \omega\right)=\widehat{\mathcal{A}}_{\ell}\left(\boldsymbol{\zeta}_{\ell}, \omega\right) \widehat{\Phi}\left(\boldsymbol{\zeta}_{\ell}, \omega\right)$ and

$$
\mathcal{H}_{\ell}\left(\frac{\boldsymbol{\theta}_{\ell}}{\mu}, \omega\right)=\int_{\widehat{\boldsymbol{c}}_{\ell}} \widehat{\mathcal{H}}_{\ell}\left(\boldsymbol{\zeta}_{\ell}, \omega\right) \exp \left(-\imath \frac{1}{\mu} \boldsymbol{\theta}_{\ell} \cdot \boldsymbol{\zeta}_{\ell}\right) d \boldsymbol{\zeta}_{\ell}
$$

If the phase profile is a quadratic fuction of $\boldsymbol{\theta}_{\ell}$, the computation of $\widehat{\mathcal{E}}_{\ell}\left(\boldsymbol{\theta}_{\ell}, \omega\right)$ is given by the following relation

$$
\widehat{\mathcal{E}}_{\ell}\left(\boldsymbol{\theta}_{\ell}, \omega\right)=\exp \left(\imath \frac{1}{\mu} \frac{\boldsymbol{\theta}_{\ell} \cdot \boldsymbol{\theta}_{\ell}}{2}\right) \mathcal{H}_{\ell}\left(\frac{\boldsymbol{\theta}_{\ell}}{\mu}, \omega\right)
$$

However, the phase profile is defined by

$$
\varphi_{\ell}\left(\boldsymbol{\xi}_{\ell}, \omega\right)=\frac{\mathcal{D}_{\ell} \sqrt{\boldsymbol{\xi}_{\ell} \cdot \boldsymbol{\xi}_{\ell}}}{1+\sqrt{1-\mathcal{D}_{\ell}^{2}}} \quad \text { with } \quad \mathcal{D}_{\ell}=\frac{\omega_{*} \sqrt{\boldsymbol{\xi}_{\ell} \cdot \boldsymbol{\xi}_{\ell}}}{\omega \sqrt{\boldsymbol{\xi}_{\ell} \cdot \boldsymbol{\xi}_{\ell}+f_{\ell}^{2}}}+\left(1-\frac{\omega_{*}}{\omega}\right) \sin \phi_{\ell}
$$

$f_{\ell}$ is the focal length, $\phi_{\ell}$ the incident beam angle and $\mathcal{N}_{\ell}\left(\boldsymbol{\beta}_{\ell}\right)$ the density associated to the focusing gratings. In practice, the second term $\left(1-\frac{\omega_{*}}{\omega}\right) \sin \phi_{\ell}$ is small compared to the first term, so that the following approximation can be used

$$
\mathcal{D}_{\ell} \simeq \frac{\omega_{*} \sqrt{\boldsymbol{\xi}_{\ell} \cdot \boldsymbol{\xi}_{\ell}}}{\omega \sqrt{\boldsymbol{\xi}_{\ell} \cdot \boldsymbol{\xi}_{\ell}+f_{\ell}^{2}}}
$$


In the general case, we can still use the preceding result obtained for the quadratic phase, by considering a modified amplitude $\mathcal{B}_{\ell}\left(\boldsymbol{\xi}_{\ell}, \omega\right)$

$$
\mathcal{B}_{\ell}\left(\boldsymbol{\xi}_{\ell}, \omega\right)=\mathcal{A}_{\ell}\left(\boldsymbol{\xi}_{\ell}, \omega\right) \exp \left[-\imath \frac{\omega}{c} \varphi_{\ell}\left(\boldsymbol{\xi}_{\ell}, \omega\right)+\imath \frac{\mu}{2} \boldsymbol{\xi}_{\ell} \cdot \boldsymbol{\xi}_{\ell}\right]
$$

Indeed

$$
\begin{aligned}
\widehat{\mathcal{E}}_{\ell}\left(\boldsymbol{\theta}_{\ell}, \omega\right) & =\int_{\mathcal{C}_{\ell}} \mathcal{A}_{\ell}\left(\boldsymbol{\xi}_{\ell}, \omega\right) \exp \left[-\imath \frac{\omega}{c} \varphi_{\ell}\left(\boldsymbol{\xi}_{\ell}, \omega\right)\right] \exp \left[-\imath\left(\boldsymbol{\theta}_{\ell} \cdot \boldsymbol{\xi}_{\ell}\right)\right] d \boldsymbol{\xi}_{\ell} \\
& =\int_{\mathcal{C}_{\ell}} \mathcal{B}_{\ell}\left(\boldsymbol{\xi}_{\ell}, \omega\right) \exp \left[-\imath \frac{\mu}{2} \boldsymbol{\xi}_{\ell} \cdot \boldsymbol{\xi}_{\ell}\right] \exp \left[-\imath\left(\boldsymbol{\theta}_{\ell} \cdot \boldsymbol{\xi}_{\ell}\right)\right] d \boldsymbol{\xi}_{\ell}
\end{aligned}
$$

For practical applications, the phase function $\varphi_{\ell}\left(\boldsymbol{\xi}_{\ell}, \omega\right)$ and the amplitude profile $\mathcal{A}_{\ell}\left(\boldsymbol{\xi}_{\ell}, \omega\right)$ are the input data. Then the electric field on the target surface is given by

$$
\boldsymbol{E}_{\ell}(t, \boldsymbol{x})=\iint_{\widehat{\boldsymbol{c}}_{\ell}} \exp \left[\imath\left(\boldsymbol{k}_{\ell}\left(\boldsymbol{\theta}_{\ell}, \omega\right) \cdot \boldsymbol{\beta}_{\ell}(\boldsymbol{x})-\omega t\right)\right] \widehat{\mathcal{E}}_{\ell}\left(\boldsymbol{\theta}_{\ell}, \omega\right) d \boldsymbol{\theta}_{\ell} d \omega
$$

where $\boldsymbol{x}$ is a point of the target surface and $\widehat{\mathcal{E}}_{\ell}\left(\boldsymbol{\theta}_{\ell}, \omega\right)$ defined as follows

$$
\widehat{\mathcal{E}}_{\ell}\left(\boldsymbol{\theta}_{\ell}, \omega\right)=\exp \left(\imath \frac{1}{\mu} \frac{\boldsymbol{\theta}_{\ell} \cdot \boldsymbol{\theta}_{\ell}}{2}\right) \int_{\widehat{\mathcal{C}}_{\ell}} \widehat{\mathcal{H}}_{\ell}\left(\boldsymbol{\zeta}_{\ell}, \omega\right) \exp \left(-\imath \frac{\boldsymbol{\theta}_{\ell} \cdot \boldsymbol{\zeta}_{\ell}}{\mu}\right) d \boldsymbol{\zeta}_{\ell}
$$

. Substituting $\mu=\frac{\omega_{*}}{c f_{\ell}}$, we obtain:

$$
\begin{aligned}
\widehat{\mathcal{H}}_{\ell}\left(\boldsymbol{\zeta}_{\ell}, \omega\right) & =-\imath \frac{2 \pi}{\mu} \exp \left(\imath \frac{1}{2 \mu} \boldsymbol{\zeta}_{\ell} \cdot \boldsymbol{\zeta}_{\ell}\right) \widehat{\mathcal{B}}_{\ell}\left(\boldsymbol{\zeta}_{\ell}, \omega\right) \\
\mathcal{B}_{\ell}\left(\boldsymbol{\xi}_{\ell}, \omega\right) & =\mathcal{A}_{\ell}\left(\boldsymbol{\xi}_{\ell}, \omega\right) \exp \left[-\imath \frac{\omega}{c} \varphi_{\ell}\left(\boldsymbol{\xi}_{\ell}, \omega\right)+\imath \frac{\mu}{2} \boldsymbol{\xi}_{\ell} \cdot \boldsymbol{\xi}_{\ell}\right]
\end{aligned}
$$

Then we can compute, for any point $\boldsymbol{x}$ of the target surface the contribution $\boldsymbol{E}_{\ell}(t, \boldsymbol{x})$ of the laser beam $\ell$ on the electric field. The total electric field is the sum of all contributions:

$$
\boldsymbol{E}(t, \boldsymbol{x})=\sum_{\ell} \boldsymbol{E}_{\ell}(t, \boldsymbol{x})
$$

\section{NumERICAL APPROXIMATION}

\subsection{Numerical scheme}

Let us consider a non overlapping finite volume decomposition of the domain $\widehat{\mathcal{C}}_{\ell}$ and describe the Fourier space by a finite number of spatial frequencies:

$$
\widehat{\mathcal{C}}_{\ell} \simeq \bigcup_{i=1}^{N k x} \bigcup_{j=1}^{N k y}\left(\widehat{\mathcal{C}}_{\ell}\right)_{i j} \quad \text { and } \quad \mathbb{R} \simeq \bigcup_{m=1}^{N w} \mathcal{I}_{m}
$$

Let us consider a piecewise constant approximation of $\widehat{\mathcal{E}}_{\ell}\left(\boldsymbol{\theta}_{\ell}, \omega\right)$ defined by

$$
\widehat{\mathcal{E}}_{\ell}\left(\boldsymbol{\theta}_{\ell}, \omega\right) \simeq \sum_{m=1}^{N w} \sum_{i=1}^{N k x} \sum_{j=1}^{N k y} \widehat{\mathcal{E}}_{\ell, i, j, m} \mathcal{X}_{i j}(\boldsymbol{\theta}) \mathcal{X}_{m}(\omega)
$$


where $\mathcal{X}_{i j}$ are characteristic functions of $\left(\widehat{\mathcal{C}}_{\ell}\right)_{i j}$, centered at $\boldsymbol{\theta}_{\ell, i, j}, \mathcal{X}_{m}(\omega)$ are characteristic functions of $\mathcal{I}_{m}$, centered at $\omega_{m}$. Then, the value $\widehat{\mathcal{E}}_{\ell, i, j, m}$ can be approximated as follows

$$
\widehat{\mathcal{E}}_{\ell, i, j, m} \simeq \exp \left(-\imath \frac{1}{\mu} \boldsymbol{\theta}_{\ell, i, j} \cdot \boldsymbol{\theta}_{\ell, i, j}\right) \mathcal{H}_{\ell}\left(\frac{\boldsymbol{\theta}_{\ell, i, j}}{\mu}, \omega_{m}\right)
$$

Therefore, we can estimate the electric field $\boldsymbol{E}_{\ell}(t, \boldsymbol{x})$ as

$$
\begin{aligned}
\boldsymbol{E}_{\ell}(t, \boldsymbol{x}) & =\iint_{\widehat{\boldsymbol{c}}_{\ell}} \exp \left[\imath\left(\boldsymbol{k}_{\ell}\left(\boldsymbol{\theta}_{\ell}, \omega\right) \cdot \boldsymbol{\beta}_{\ell}(\boldsymbol{x})-\omega t\right)\right] \widehat{\mathcal{E}}_{\ell}\left(\boldsymbol{\theta}_{\ell}, \omega\right) d \boldsymbol{\theta}_{\ell} d \omega \\
& \simeq \sum_{m=1}^{N w} \sum_{i=1}^{N k x} \sum_{j=1}^{N k y} \widehat{\mathcal{E}}_{\ell, i, j, m} \int_{\mathcal{I}_{m}} \int_{\left(\widehat{\boldsymbol{c}}_{\ell}\right)_{i j}} \exp \left[\imath\left(\boldsymbol{k}_{\ell}\left(\boldsymbol{\theta}_{\ell}, \omega\right) \cdot \boldsymbol{\beta}_{\ell}(\boldsymbol{x})-\omega t\right)\right] \boldsymbol{\theta}_{\ell} d \omega \\
& \simeq \sum_{m=1}^{N w} \sum_{i=1}^{N k x} \sum_{j=1}^{N k y} \widehat{\mathcal{E}}_{\ell, i, j, m} \mathcal{Q}_{\ell, i, j, m}(\boldsymbol{x}, t)
\end{aligned}
$$

with

$$
\mathcal{Q}_{\ell, i, j, m}(\boldsymbol{x}, t)=\int_{\mathcal{I}_{m}} \int_{\left(\widehat{\boldsymbol{c}}_{\ell}\right)_{i j}} \exp \left[\imath\left(\boldsymbol{k}_{\ell}\left(\boldsymbol{\theta}_{\ell}, \omega\right) \cdot \boldsymbol{\beta}_{\ell}(\boldsymbol{x})-\omega t\right)\right] \boldsymbol{\theta}_{\ell} d \omega
$$

where $\boldsymbol{\beta}_{\ell}(\boldsymbol{x})=\boldsymbol{x}-\boldsymbol{x}_{\ell}$ and $\boldsymbol{\theta}_{\ell}, \omega$ are as defined in the previous section. In order to obtain a simple and accurate estimation of $\mathcal{Q}_{\ell, i, j, m}$, let us perform a Taylor expansion of $k_{z, \ell}\left(\boldsymbol{\theta}_{\ell}, \omega\right)$ at the point $\left(\left(\boldsymbol{\theta}_{\ell}\right)_{i, j}, \omega_{m}\right)$ :

$$
k_{z, \ell}\left(\boldsymbol{\theta}_{\ell}, \omega\right)=k_{z, \ell}\left(\left(\boldsymbol{\theta}_{\ell}\right)_{i j}, \omega_{m}\right)-\frac{\left(\boldsymbol{\theta}_{\ell}\right)_{i j} \cdot\left(\boldsymbol{\theta}_{\ell}-\left(\boldsymbol{\theta}_{\ell}\right)_{i j}\right)-\frac{\omega_{m}}{c^{2}}\left(\omega-\omega_{m}\right)}{k_{z, \ell}\left(\left(\boldsymbol{\theta}_{\ell}\right)_{i j}, \omega_{m}\right)}+O\left(\delta \boldsymbol{\theta}_{\ell}^{2}\right)+O\left(\delta \omega^{2}\right)
$$

Then, after straightforward algebra, we get the following approximation for $\phi\left(\boldsymbol{\theta}_{\ell}, \omega\right)=\boldsymbol{k}_{\ell}\left(\boldsymbol{\theta}_{\ell}, \omega\right) \cdot \boldsymbol{\beta}_{\ell}(\boldsymbol{x})-\omega t$ :

$$
\phi\left(\boldsymbol{\theta}_{\ell}, \omega\right) \simeq \phi\left(\boldsymbol{\theta}_{i j}, \omega_{m}\right)+\boldsymbol{\alpha}_{i j} \cdot\left(\boldsymbol{\theta}_{\ell}-\boldsymbol{\theta}_{i j}\right)-\alpha_{m}\left(\omega-\omega_{m}\right)
$$

where, for clarity, the index $\ell$ has been removed $\left(\boldsymbol{\theta}_{i j} \equiv\left(\boldsymbol{\theta}_{\ell}\right)_{i j}\right)$,

$$
\boldsymbol{\alpha}_{i j}=\boldsymbol{\xi}_{\ell}(\boldsymbol{x})-\frac{z_{\ell}(\boldsymbol{x}) \boldsymbol{\theta}_{i j}}{k_{0, \ell}\left(\boldsymbol{\theta}_{i j}, \omega_{m}\right)}=\left(\begin{array}{c}
\alpha_{i j}^{x} \\
\alpha_{i j}^{y}
\end{array}\right) \quad \text { and } \quad \alpha_{m}=t-\frac{\omega_{m} z_{\ell}(\boldsymbol{x})}{c^{2} k_{z, \ell}\left(\boldsymbol{\theta}_{i j}, \omega_{m}\right)}
$$

For an uniform mesh $\mathcal{I}_{m}=\left[\omega_{m}-\frac{\delta \omega}{2}, \omega_{m}+\frac{\delta \omega}{2}\right]$ and

$$
\left(\widehat{\boldsymbol{c}}_{\ell}\right)_{i j} \equiv\left[\left(\theta_{1, \ell}\right)_{i j}-\frac{\delta \theta_{1, \ell}}{2}, \quad\left(\theta_{1, \ell}\right)_{i j+\frac{\delta \theta_{1, \ell}}{2}}\right] \times\left[\left(\theta_{2, \ell}\right)_{i j}-\frac{\delta \theta_{2, \ell}}{2}, \quad\left(\theta_{2, \ell}\right)_{i j}+\frac{\delta \theta_{2, \ell}}{2}\right]
$$


The value of $\mathcal{Q} \equiv \mathcal{Q}_{\ell, i, j, m}(\boldsymbol{x}, t)$ is computed as

$$
\begin{aligned}
\mathcal{Q} & =\exp \left[\imath \phi\left(\boldsymbol{\theta}_{i j}, \omega_{m}\right)\right] \int_{-\frac{\delta \omega}{2}}^{\frac{\delta \omega}{2}} \int_{-\frac{\delta \theta_{x}}{2}}^{+\frac{\delta \theta_{x}}{2}} \int_{-\frac{\delta \theta_{y}}{2}}^{+\frac{\delta \theta_{y}}{2}} \exp \left[\imath\left(\boldsymbol{\alpha}_{i j} \cdot \boldsymbol{\theta}_{i j}-\alpha_{m} \omega\right)\right] d \boldsymbol{\theta} d \omega \\
& =\exp \left[\imath \boldsymbol{k}_{\ell}\left(\boldsymbol{\theta}_{i j}, \omega_{m}\right) \cdot \boldsymbol{\beta}_{\ell}(\boldsymbol{x})\right] \exp \left[-\imath \omega_{m} t\right] \mathcal{G}_{\ell, h}\left(t, \boldsymbol{x}, \omega_{m}, \boldsymbol{\theta}_{i j}\right) \\
& =\exp \left[\imath\left(\boldsymbol{\theta}_{i j} \cdot \boldsymbol{\xi}_{\ell}(\boldsymbol{x})+k_{z, \ell} z_{\ell}\right)\right] \exp \left[-\imath \omega_{m} t\right] \mathcal{G}_{\ell, h}\left(t, \boldsymbol{x}, \omega_{m}, \boldsymbol{\theta}_{i j}\right)
\end{aligned}
$$

with

$$
\mathcal{G}_{\ell, h}\left(t, \boldsymbol{x}, \omega_{m}, \boldsymbol{\theta}_{i j}\right)=\frac{2 \sin \left(\alpha_{i j}^{x} \frac{\delta \theta_{x}}{2}\right)}{\alpha_{i j}^{x}} \frac{2 \sin \left(\alpha_{i j}^{y} \frac{\delta \theta_{y}}{2}\right)}{\alpha_{i j}^{y}} \frac{2 \sin \left(\alpha_{m} \frac{\delta \omega}{2}\right)}{\alpha_{m}}
$$

Then the electric field is approximated by

$$
\boldsymbol{E}_{\ell}(t, \boldsymbol{x}) \simeq \sum_{m=1}^{N w} \sum_{i=1}^{N k x} \sum_{j=1}^{N k y} \widehat{\mathcal{E}}_{\ell, i, j, m} \mathcal{Q}_{\ell, i, j, m}(\boldsymbol{x}, t) \simeq \sum_{m=1}^{N w} \widehat{\boldsymbol{E}}_{\ell}\left(\omega_{m}, \boldsymbol{x}\right) \exp \left[-\imath \omega_{m} t\right]
$$

where it is assumed that $\mathcal{G}_{\ell, h}\left(t, \boldsymbol{x}, \omega_{m}, \boldsymbol{\theta}_{i j}\right) \equiv \mathcal{G}_{\ell, h}\left(\boldsymbol{x}, \omega_{m}, \boldsymbol{\theta}_{i j}\right)$ and

$$
\widehat{\boldsymbol{E}}_{\ell}\left(\omega_{m}, \boldsymbol{x}\right) \simeq \sum_{i=1}^{N k x} \sum_{j=1}^{N k y} \widehat{\mathcal{E}}_{\ell, i, j, m} \mathcal{G}_{\ell, h}\left(\boldsymbol{x}, \omega_{m}, \boldsymbol{\theta}_{i j}\right) \exp \left[\imath\left(\boldsymbol{\theta}_{i j} \cdot \boldsymbol{\xi}_{\ell}(\boldsymbol{x})+k_{z, \ell} z_{\ell}\right)\right]
$$

Indeed, when $\delta \omega$ and $\delta \boldsymbol{\theta}$ are small enough, we have $\mathcal{G}_{\ell, h}\left(t, \boldsymbol{x}, \omega_{m}, \boldsymbol{\theta}_{i j}\right) \simeq \delta \omega \delta \boldsymbol{\theta}$. Another assumption that is consistent with target applications is that

$$
\mathcal{A}_{\ell}\left(\boldsymbol{\xi}_{\ell}, \omega\right) \equiv A_{\ell}\left(\boldsymbol{\xi}_{\ell}\right) \widehat{\mathcal{T}}_{\ell}(\omega)
$$

where $\widehat{\mathcal{T}}_{\ell}(\omega)$ is the Fourier transform of a given temporal profile $\mathcal{T}_{\ell}(t)$ and $A_{\ell}\left(\boldsymbol{\xi}_{\ell}\right)$ is the given spatial profile.

The main steps of the numerical scheme are thus (Software Design):

- Compute the vector $\underline{A_{\ell}}\left(\underline{\boldsymbol{\xi}_{\ell}}\right)$ that components are $A_{\ell}\left(\left(\boldsymbol{\xi}_{\ell}\right)_{i, j}\right)$, for $i=1, \cdots, N_{x}$ and $j=1, \cdots, N_{y}$.

- Perform fast Fourier transform $\mathcal{T}_{\ell}(t)$ to obtain $\widehat{\mathcal{T}}_{\ell}\left(\omega_{m}\right)$ for $m=1, \cdots, N w$

- For any $\omega_{m}$

(1) Compute $\underline{\mathcal{A}_{\ell}}\left(\underline{\boldsymbol{\xi}_{\ell}}, \omega_{m}\right):=\underline{A_{\ell}}\left(\underline{\xi_{\ell}}\right) \widehat{\mathcal{T}}_{\ell}\left(\omega_{m}\right)$

(2) Add the contribution of the phase in the physical space

$$
\underline{\mathcal{B}_{\ell}}\left(\underline{\boldsymbol{\xi}_{\ell}}, \omega_{m}\right):=\underline{\mathcal{A}_{\ell}}\left(\underline{\boldsymbol{\xi}_{\ell}}, \omega_{m}\right) \exp \left[-\imath \frac{\omega_{m}}{c} \underline{\varphi_{\ell}}\left(\underline{\boldsymbol{\xi}_{\ell}}, \omega_{m}\right)+\imath \frac{\mu}{2} \underline{\boldsymbol{\xi}_{\ell}} \cdot \underline{\boldsymbol{\xi}_{\ell}}\right]
$$

(3) Perform fast Fourier transform $\underline{\mathcal{B}_{\ell}}\left(\underline{\boldsymbol{\xi}_{\ell}}, \omega_{m}\right)$ to obtain $\underline{\widehat{\mathcal{B}_{\ell}}}\left(\underline{\boldsymbol{\zeta}_{\ell}}, \omega_{m}\right)$

(4) Add the contribution of the phase in the phase space :

$$
\underline{\widehat{\mathcal{H}}_{\ell}}\left(\underline{\boldsymbol{\zeta}_{\ell}}, \omega_{m}\right)=-\imath \frac{2 \pi}{\mu} \underline{\widehat{\mathcal{B}_{\ell}}}\left(\underline{\boldsymbol{\zeta}_{\ell}}, \omega_{m}\right) \exp \left(\imath \frac{1}{2 \mu} \underline{\boldsymbol{\zeta}_{\ell}} \cdot \underline{\boldsymbol{\zeta}_{\ell}}\right)
$$

(5) Perform fast Fourier transform $\underline{\widehat{\mathcal{H}}_{\ell}}\left(\underline{\boldsymbol{\zeta}_{\ell}}, \omega_{m}\right)$ to obtain $\underline{\mathcal{H}_{\ell}}\left(\frac{\underline{\boldsymbol{\theta}}}{\mu}, \omega_{m}\right)$

$$
\mathcal{H}_{\ell}\left(\frac{\boldsymbol{\theta}_{\ell, k x, k y}}{\mu}, \omega_{m}\right)=\int_{\widehat{\boldsymbol{c}}_{\ell}} \widehat{\mathcal{H}}_{\ell}\left(\boldsymbol{\zeta}_{\ell}, \omega\right) \exp \left(-\imath \frac{\boldsymbol{\theta}_{\ell, k x, k y}}{\mu} \cdot \boldsymbol{\zeta}_{\ell}\right) d \boldsymbol{\zeta}_{\ell}
$$


(6) Modification of the spectral profile

$$
\widehat{\mathcal{E}}_{\ell, m}=\exp \left(\imath \frac{1}{\mu} \frac{\underline{\boldsymbol{\theta}}_{\ell} \cdot \underline{\boldsymbol{\theta}}_{\ell}}{2}\right) \underline{\mathcal{H}_{\ell}}\left(\frac{\underline{\boldsymbol{\theta}}_{\ell}}{\mu}, \omega_{m}\right)
$$

(7) Electric field on the focus plane is defined by a finite number of points $\left(N f_{x} \times N f_{y}\right)$ :

$$
\widehat{\underline{\boldsymbol{E}}}_{\ell}\left(\omega_{m}, \underline{\boldsymbol{x}}\right) \equiv \widehat{\boldsymbol{E}}_{\ell}\left(\omega_{m}, \boldsymbol{x}_{i x, i y}\right) \quad \text { for } \quad i x=1, \cdots, N f_{x} \quad \text { and } \quad i y=1, \cdots, N f_{y}
$$

where

$$
\widehat{\boldsymbol{E}}_{\ell}\left(\omega_{m}, \boldsymbol{x}\right) \simeq \sum_{k x=1}^{N k x} \sum_{k y=1}^{N k y} \widehat{\mathcal{E}}_{\ell, k x, k y, m} \mathcal{G}_{\ell, h}\left(\boldsymbol{x}, \omega_{m}, \boldsymbol{\theta}_{k x, k y}\right) \exp \left[\imath\left(\boldsymbol{\theta}_{k x, k y} \cdot \boldsymbol{\xi}_{\ell}(\boldsymbol{x})+k_{z, \ell} z_{\ell}\right)\right]
$$

(8) Total field generated by the sum of laser spots:

$$
\widehat{\underline{\boldsymbol{E}}}\left(\omega_{m}, \underline{\boldsymbol{x}}\right):=\sum_{\ell} \underline{\underline{\boldsymbol{E}}}_{\ell}\left(\omega_{m}, \underline{\boldsymbol{x}}\right)
$$

- Perform fast Fourier transform $\underline{\widehat{\boldsymbol{E}}}\left(\omega_{m}, \underline{\boldsymbol{x}}\right)$ to obtain $\underline{\widehat{\boldsymbol{E}}}(t, \underline{\boldsymbol{x}})$. For this last step we need to save an array of the size $N w \times N f_{x} \times N f_{y}$

\subsection{Parallel Strategy}

Our target application is the "Laser Mega Joule" (LMJ). In this context, the input data are defined with size of order $N w=O\left(10^{3}\right), N k x=O\left(10^{3}\right), N k y=O\left(10^{3}\right)$ and $N l=O(10)$. If the required resolution on the target surface is $N f x \times N f y$ then the computation of the focal spot is achieved with a total number of operations of order $N f x \times N f y \times O\left(10^{13}\right)$. Therefore, even for a coarse resolution $(N f x=N f y=100)$ of the focal spot, we need many days of CPU time on a tera computer to perform computations. As tera-computers are always parallel, we need to distribute the total charge on the set of processors such as to efficiently use parallel architectures.

The first level of parallel strategy consists in the domain decomposition of the focal spot. Let us denote by $\Omega$ the focal spot and use the following decomposition:

$$
\Omega=\bigcup_{g=0}^{N g} \Omega_{g} \quad \text { and each group }(\mathrm{g}) \text { of processes will compute only } \quad \underline{\widehat{E}}_{g}(t, \underline{\boldsymbol{x}}) \equiv \underline{\widehat{E}}\left(t, \underline{\boldsymbol{x}} \in \Omega_{g}\right)
$$

where $N g$ is the number of group at this level. At this level we do not need any exchange of messages between groups. Then, if the load balance is achieved with one process per group, the efficiency is optimal.

If the computation of $\widehat{\underline{E}}_{g}(t, \underline{\boldsymbol{x}})$ can not be achieved on one process, another step of the parallel strategy is performed by a domain decomposition of the domain associated to the initial laser beams :

$$
\mathcal{C}_{\ell}=\bigcup_{e=0}^{N e}\left(\mathcal{C}_{\ell}\right)_{e} \quad \text { and } \quad \widehat{\mathcal{C}}_{\ell}=\bigcup_{e=0}^{N e}\left(\widehat{\mathcal{C}}_{\ell}\right)_{e}
$$

If the two levels are used, a process will be associated to a group $g$ and will compute the contribution $\underline{\boldsymbol{E}}_{g, e}(t, \underline{\boldsymbol{x}})$ associated to $\left(\mathcal{C}_{\ell}\right)_{e}$ and $\left(\widehat{\boldsymbol{C}}_{\ell}\right)_{e}$, such as

$$
\underline{\underline{\boldsymbol{E}}}_{g}(t, \underline{\boldsymbol{x}})=\sum_{e \in g} \underline{\widehat{\boldsymbol{E}}}_{g, e}(t, \underline{\boldsymbol{x}})
$$


Computation of $\widehat{\underline{\boldsymbol{E}}}_{g, e}(t, \underline{\boldsymbol{x}})$ and $\underline{\boldsymbol{E}}_{g}(t, \underline{\boldsymbol{x}})$ needs to exchange messages between process of the same group. This is achieved by the use of Message Passing Interface (MPI) and a parallel version of the FFTW (Fast Fourier Transform in the West) [4].

\section{Application}

In order to test the possibilities of the method described above, several computations have been run on the machines Titane and Platine at the CCRT center near Paris. For any computation, the data are the electromagnetic field, corresponding to a quad, at the entrance of the experimental chamber provided by the MIRÓ code [3]. It takes into account all components of the LMJ chain, including the phase plates. But it does not account for the focusing gratings and their effect on the signal phase. The effects of the focusing gratings are included in the data as it was explained in the above sections.

The first type of calculation was done for a monochromatic quad at the wavelength of 351 nanometer whose direction corresponds to a value of $49^{\circ}$ for the angle $\psi$ and to $288^{\circ}$ for the angle $\theta$. The field is calculated at the Hohlraum entrance located at the target center with the normal directed at $\psi=0$ and $\theta=0$. Two calculations have been done to compute the electric field on $1024 \times 1024$ points in a square of $125 \mu$ centered on the middle of the entrance face of Hohlraum. The first calculation uses data defined on a 1024x1024 mesh and a 2048x2048 mesh has been used for the second computation. The field amplitude is shown respectively on figures 5 and 6 . The results are almost the same. This as well as other results show that initial data defined on a 1024x1024 mesh are generally enough. The $2048 \times 2048$ mesh should only be used for very precise results. Those computations ran respectively 28 minutes and 125 minutes on 64 cores, 8 nodes (2 Intelß) Nehalem, Quadri-Core, 2.93 GHz, 24 Go) of Titane.

The second type of calculation presents an example with a large number of monochromatic beams and very fine meshes. The data are defined on a 2048x2048 mesh, while the solution is computed on a 2048x2048 mesh. Figure 7 shows thus the electric field amplitude at the Hohlraum entrance for 120 beams (30 quads). This computation ran 21 hours on 1936 cores, 242 nodes (4 Intel@ Itanium®II, Dual-Core, $1.6 \mathrm{GHz}, 128 \mathrm{Go}$ ) of Platine. With this large number of beams the focal spot has a form of a circle. On the other hand despite the large number of points, the mesh is not fine enough to describe the structure of the hot spots. This was not the case for a quad. Thus a superposition of a large number of beams modifies seriously the hot spot structure. Another computation on a smaller surface would be needed to observe this structure.

Figures from 8 to 11 illustrate the possibility to calculate the electric field on any surface. They represent the distribution of intensity created by a quad on the inner surface of a Hohlraum assuming propagation in vacuum. This surface is parameterized by the projection on the cylinder $\mathrm{z}$ axis which gives the ordinate and by the azimuthal angle which defines the abscissa. Figure 8 corresponds to a cone (ten quads) entering with an angle $\psi=33.2^{\circ}$ (see figure 3). Similar distributions for the angles of $49^{\circ}$ and $59^{\circ}$ are presented on figures 9 and 10. While in figure 10 the focal spots of the four beams of a quad are virtually superimposed, they seem to begin to separate for the other angles. This affects the focal spot in the direction of the axis of Hohlraum. On the other hand, the focal spots are spread over almost sixty degrees, which ensures a good recovery of focal spots for the ten quads of a cone.The figure 11 shows the surface illuminated by the three cones. The advantage of this approach is that we need to compute the field for only one quad per cone. The fields induced by the other quads are simply obtained with a rotation.

Finally to illustrate how the optical smoothing works two runs with time variation of the laser beams have been performed. One for a surface containing the focus spot, and the other for a smaller surface inside this spot. For both runs the ten quads from the cone with angle $\psi=33.2^{\circ}$ have been considered. The smoothing of the laser beams is obtained by multiplication of the input data with a phase term equal to $\exp \left[15 i \sin \left(\omega_{s} \cdot t\right)\right]$ where $\omega_{s}$ is the smoothing frequency corresponding to the introduced spectral width. The energy flux through the Hohlraum entrance is plotted in the right of figures 12 and 13 . It should be compared with the left parts of those figures which corresponds to the same results for a monochromatic beam.Each computation in the time 
domain was equivalent to 28 monochromatic runs and took 7 hours on $28 x 196$ cores from Titane. These results show that the optical smoothing significantly improves the homogeneity of the laser irradiation of the focal spot. 


\section{Conclusion}

The ultimate goal of this work is to obtain a spatial and temporal description of the total field created on the target in the LMJ laser installation. We developed the methodology and presented the first results for a large variety of configurations. Monochromatic waves as well as pulses have been considered. The computation time is, as expected, large but remains realistic. If for one beam or a quad, a single computation is necessary to obtain the focus spot size as well as its structure, this is no longer true for a large number of beams. In this case indeed, the hot spots are too small and a second computation is needed to study the hot spots structure. This numerical tool should be useful for laser specialists as well as for plasma specialists, and it could become a meeting point between both groups. It will help to understand the influence of many parameters from the laser chain and to define the boundary conditions for computations of the plasma evolution in the Hohlraum.

\section{REFERENCES}

[1] R. Courant und D. Hilbert, "Methoden der Mathematischen Physik II", Springer Verlag (1968).

[2] A. Kastler, "Cours de physique générale, optique", éditions Masson (1965).

[3] O. Morice, "MIRÓ a complete modeling and software for pulse amplification and propagation in high power laser systems", Optical Engineering 6, 1530 (2003).

[4] M. Frigo and S.-G. Johnson "The Design and Implementation of FFTW3", Proceedings of the IEEE 93, 2, 216-231 (2005).

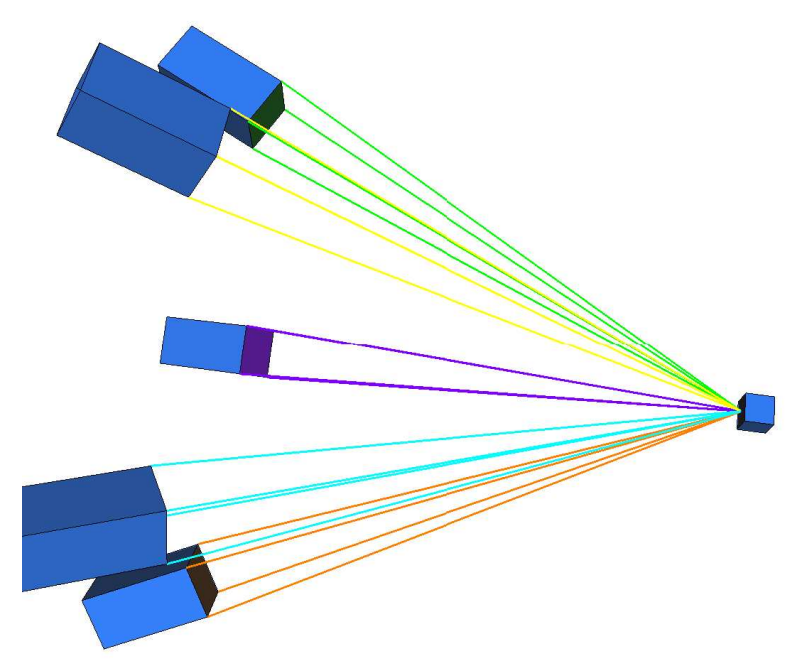

FiguRE 3. LMJ case: incoming beams (left boxes), focal spot (right box). Incoming waves are boxes of $1.2 \mathrm{~m} \times 1.2 \mathrm{~m}$ and the focal spot is a plane of $1.2 \mathrm{~mm} \times 1.2 \mathrm{~mm}$. The focal length is $f_{\ell}=8 m$ 


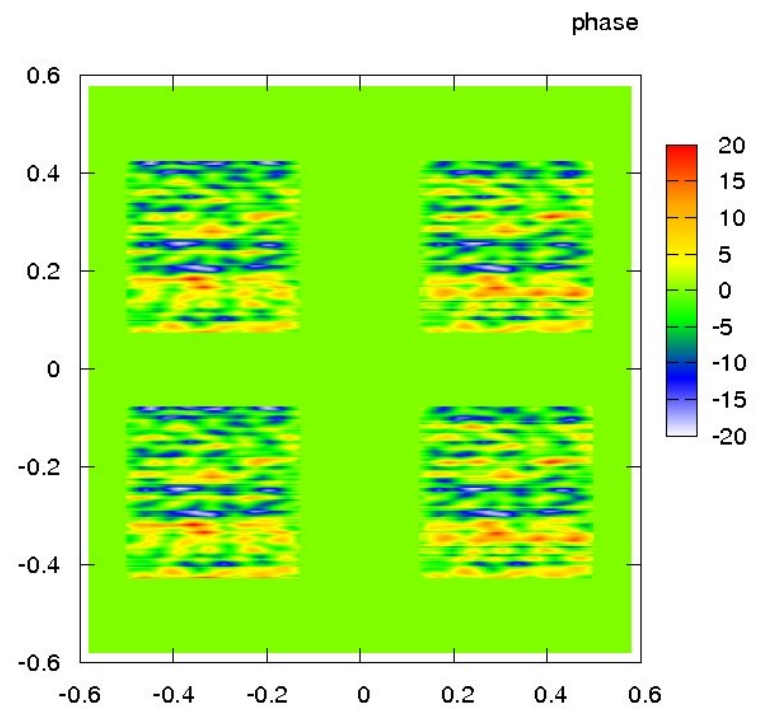

Figure 4. The phase of the incoming beam for a fixed frequency. The length unit is the meter, the phase unit is the radian. Resolution of $2048 \times 2048$
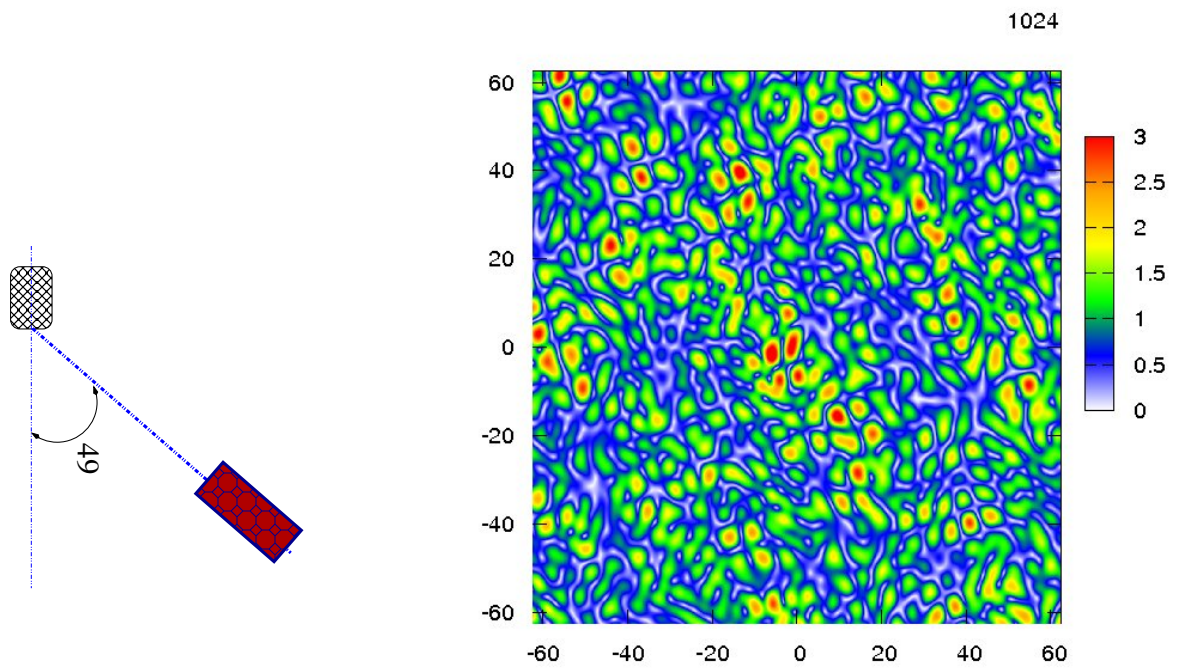

FiguRE 5. Zoom on the computed focal spot with the resolution $1024 \times 1024:\|\boldsymbol{E}(\boldsymbol{x}, T)\|$. The length unit is the micrometer. The electric field unit is arbitrary. 


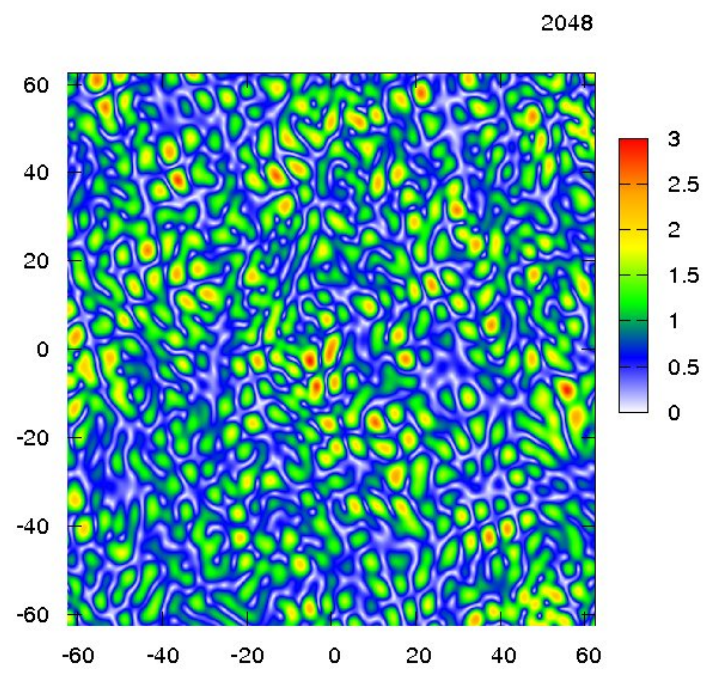

Figure 6. Zoom on the computed focal spot with the resolution $2048 \times 2048:\|\boldsymbol{E}(\boldsymbol{x}, T)\|$. The length unit is the micrometer. The electric field unit is arbitrary.
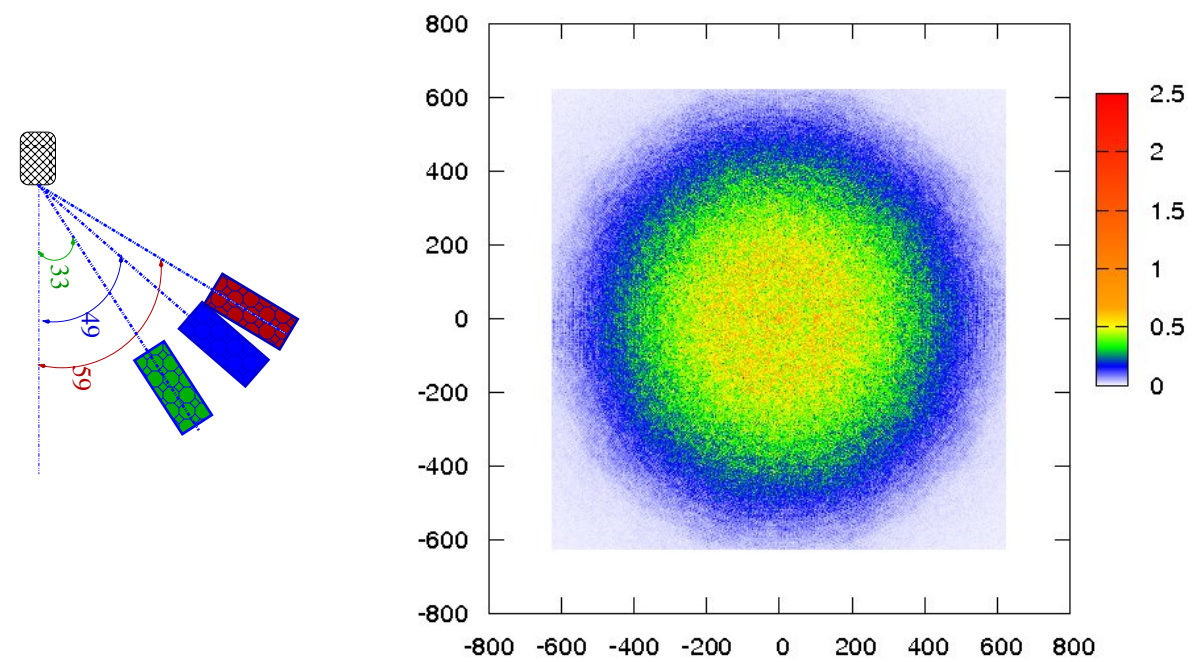

Figure 7 . Computed focal spot with the resolution $2048 \times 2048:\|\boldsymbol{E}(\boldsymbol{x}, T)\|$. The length unit is the micrometer. The electric field unit is arbitrary. 

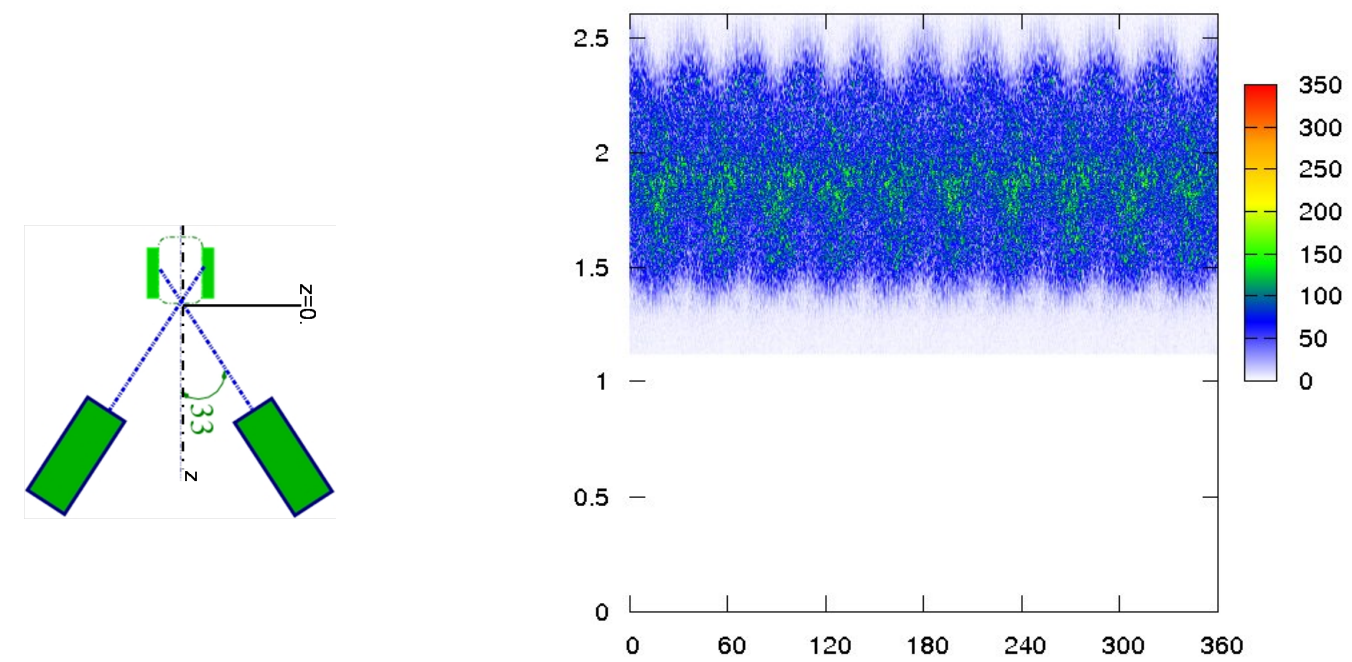

Figure 8. Computed spot on the Hohlraum's inner surface, first cone : $\|\boldsymbol{E}(\boldsymbol{x}, \omega)\|$. The length unit is the millimeter. The electric field unit is arbitrary. The abscissa is the azimuthal angle in degrees.

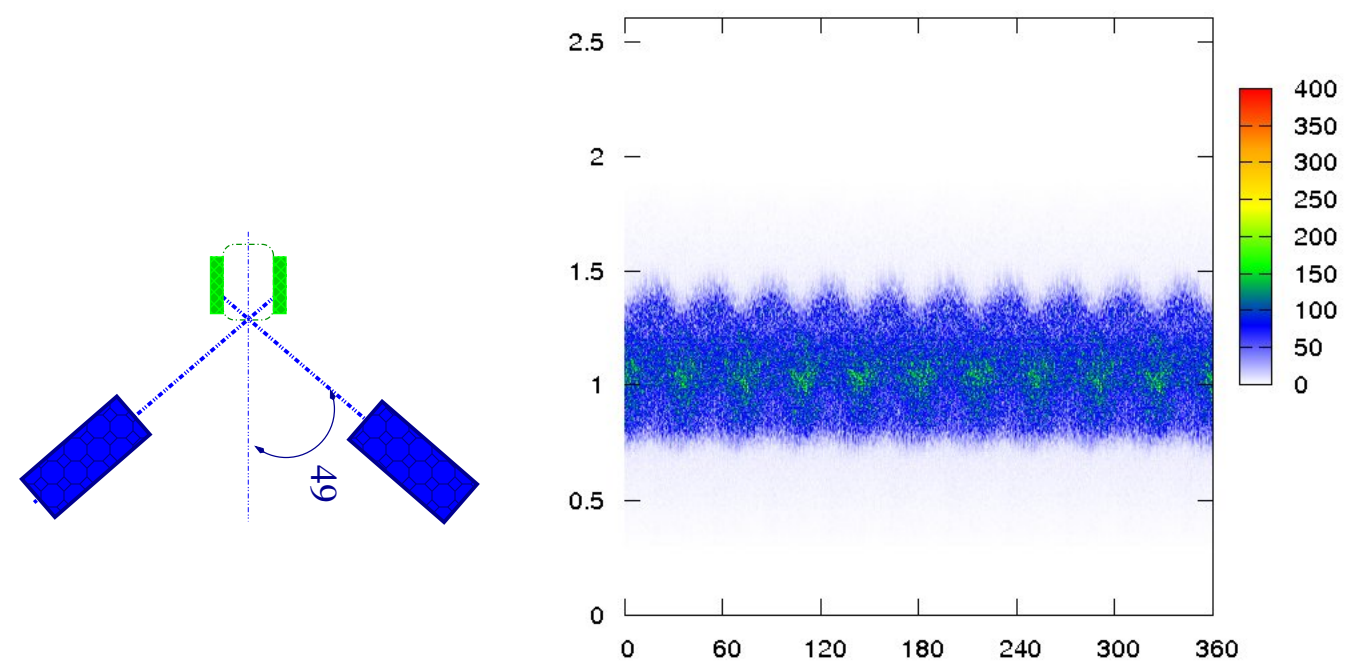

Figure 9. Computed spot on the Hohlraum's inner surface, second cone : $\|\boldsymbol{E}(\boldsymbol{x}, \omega)\|$. The length unit is the millimeter. The electric field unit is arbitrary. The abscissa is the azimuthal angle in degrees. 


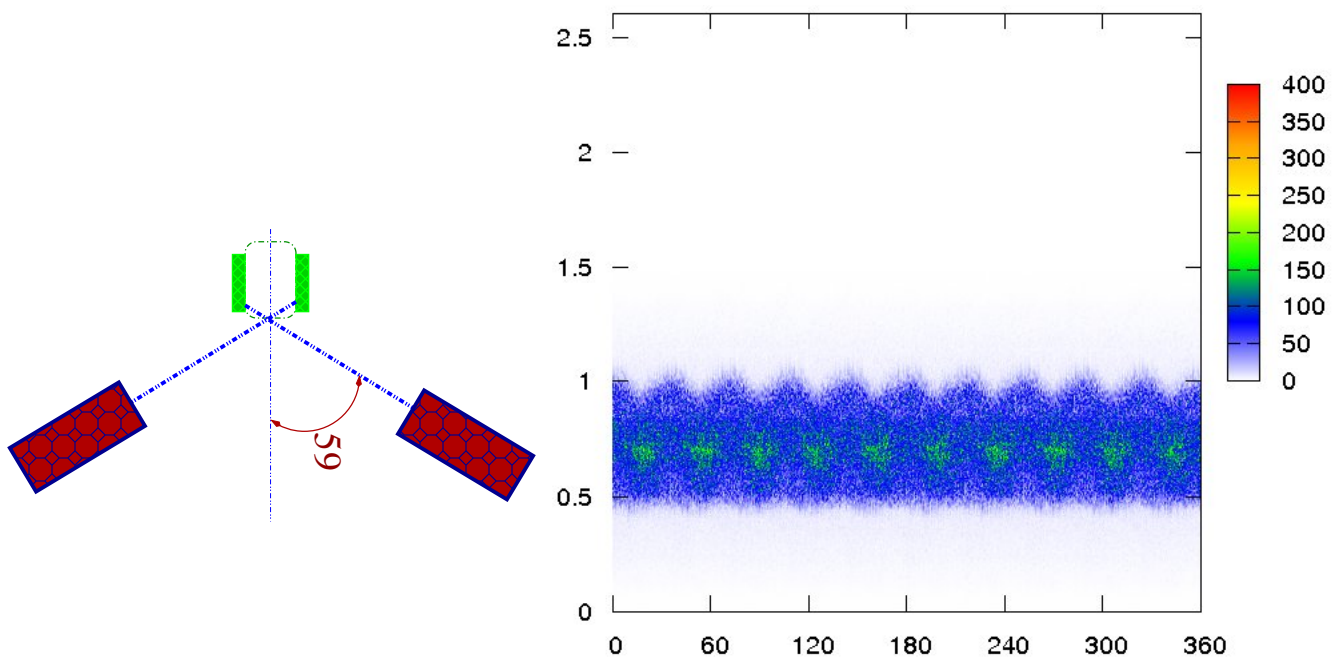

Figure 10. Computed spot on the Hohlraum's inner surface, third cone : $\|\boldsymbol{E}(\boldsymbol{x}, \omega)\|$. The length unit is the millimeter. The electric field unit is arbitrary. The abscissa is the azimuthal angle in degrees.

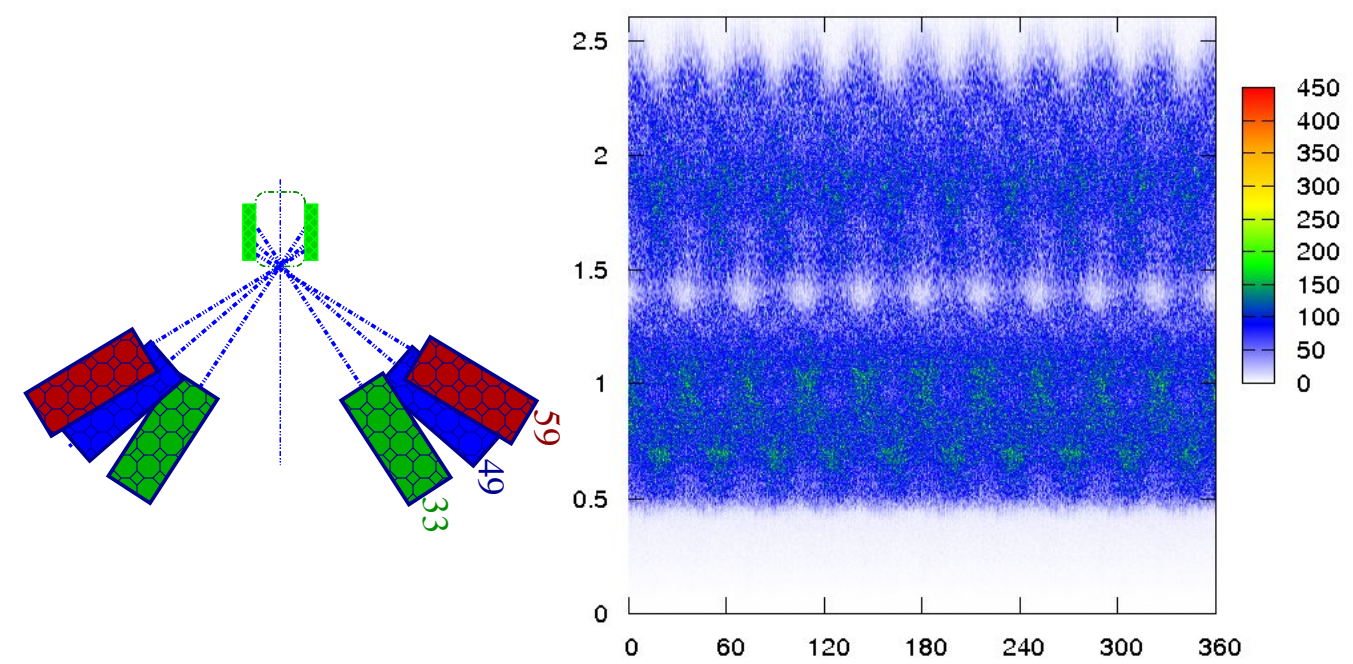

Figure 11. Computed spot on the Hohlraum's inner surface, three cones : $\|\boldsymbol{E}(\boldsymbol{x}, \omega)\|$. The length unit is the millimeter. The electric field unit is arbitrary. The abscissa is the azimuthal angle in degrees. 

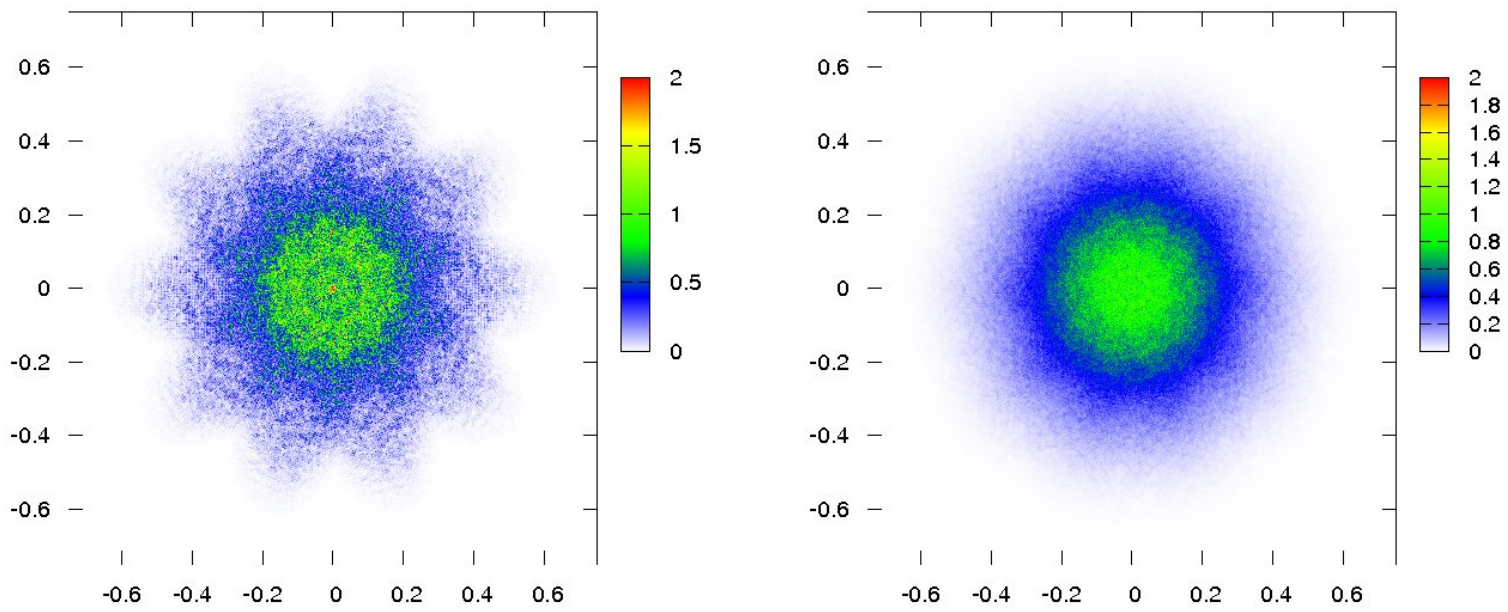

Figure 12. Computed Energy flux through the Hohlraum's aperture, with (right) and without (left) smoothing: $\|F(\boldsymbol{x})\|$. The length unit is the millimeter. The electric field unit is arbitrary.
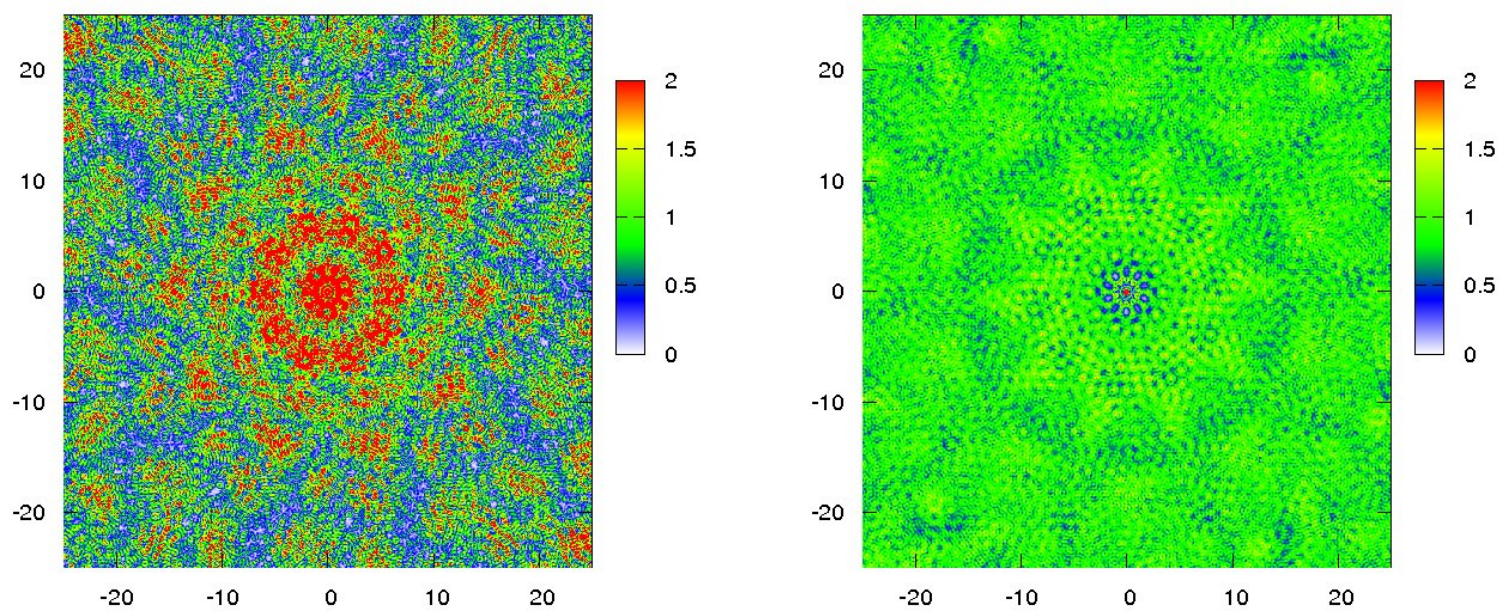

Figure 13. Computed Energy flux through the Hohlraum's aperture, with (right) and without (left) smoothing : $\|F(\boldsymbol{x})\|$. The length unit is the micrometer. The electric field unit is arbitrary. 